\title{
Hydrolysis of Anilides
}

\section{II.* Hydrolysis of Trifluoro- and Trichloroacetanilide by Hydroxyl Ions and by Some Other Catalysts}

\author{
SVENO. ERIKSSON and CHRISTIA NE HOLST
}

Department of Chemistry, Kungl. Farmaceutiska Institutet, Stockholm, Sweden

\begin{abstract}
The $\mathrm{p} K_{\mathrm{a}}$ values of trifluoroacetanilide $\left(\mathrm{C}_{6} \mathrm{H}_{5} \mathrm{NHCOCF}_{3}\right)$ and trichloroacetanilide $\left(\mathrm{C}_{6} \mathrm{H}_{5} \mathrm{NHCOCCl}_{3}\right)$ have been determined and, using a pH-stat method, the compounds have been hydrolysed at $25^{\circ} \mathrm{C}$ in a constant ionic medium at hydroxyl ion concentrations ranging from 1 to about $10^{-6} \mathrm{M}$. The curves obtained when the observed pseudo first-order rate constants ( $\left.k_{\mathrm{obs}}\right)$ or their logarithms are plotted against hydroxyl ion concentrations or their logarithms (so-called pH-rate profiles) show a limiting value of $k_{\text {obs }}$ at high pH values and the curves are of the same shape for both compounds. The mechanism of hydroxyl ion catalysis is discussed in some detail, and on the basis of comparisons of rate parameters of the two compounds, the hypothesis of dipolar anion formation preceding breakdown to products is discussed.

The rate of hydrolysis is strongly enhanced by the presence of very small concentrations of some species, such as the hydrogen carbonate and dihydrogen phosphate ions. It is shown that this effect can be interpreted as catalytic breakdown of an intermediate between substrate and hydroxyl ions. Hydroxyl ions also effect breakdown of the intermediate but are less efficient than the anions mentioned. From the equations derived, it is apparent that at a given $\mathrm{pH}$ all species acting in the breakdown of the intermediate alone, are expected to give the same limiting value of $k_{\text {obs. }}$. At pH values smaller than $\mathrm{p} K_{\mathrm{a}}$ this limiting value of the rate constant for both compounds is about 40 times larger than its value in the absence of such catalysts.
\end{abstract}

A nilides are generally very stable towards alkaline hydrolysis, but compounds containing strongly electron attracting groups in the acylic part of the molecule are hydrolysed fairly rapidly. In an often cited paper, Biechler and Taft Jr.1 reported on the alkaline hydrolysis of trifluoroacetanilide in water and in aqueous dioxane at $25.5^{\circ} \mathrm{C}$ and concentrations of hydroxyl ions ranging from 0.006 to $0.5 \mathrm{M}$. They found that the rate was not proportional

* Part I: see Ref. 9.

Acta Chem. Scand. 20 (1966) No. 7 
to the hydroxyl ion concentrations, and the relation between rate and $\mathrm{pH}$ was explained on the basis of ionization of trifluoroacetanilide at high $\mathrm{pH}$ values and stability of the corresponding base towards alkaline hydrolysis. The kinetic data gave a $\mathrm{p} K_{\mathrm{a}}$ value of 11.9 . Bourne et al. ${ }^{2}$ found that trifluoroacetanilide in aqueous alcoholic solution could be titrated against $0.1 \mathrm{M}$ sodium hydroxide with an end point around $\mathrm{pH} 11-11.5$, which shows that the compound has a $\mathrm{p} K_{\mathrm{a}}$ value much smaller than 11.9. When the investigation here reported was partly completed, a paper by Mader ${ }^{3}$ appeared where he reports a $\mathrm{p} K_{\mathrm{a}}$ of 9.5 at $25^{\circ} \mathrm{C}$. He suggests a mechanism of hydroxyl ion catalysis which is principally accepted by us and discussed here in greater detail. The curve giving the relation between $\log k_{\mathrm{obs}}$ and $\mathrm{pH}$ for trifluoroacetanilide (the pH-rate profile) obtained by Mader is at the higher hydroxyl ion concentrations in good agreement with that reported in this paper. At the lower hydroxyl ion concentrations, however, the agreement is less good. Mader obtained values for the rate parameters with the aid of computer analysis. It is shown that from the data now obtained, the parameters necessary for construction of a $\mathrm{pH}$-rate profile can easily be evaluated without making use of computer analysis.

Except for the paper by Mader, catalysis by buffer species has, as far as we know, not been reported in anilide hydrolysis.* In his experiments, Mader used four different buffer systems, namely mono-, di-, and triethanolamine and morpholine. For the three ethanolamines, he found that at constant $\mathrm{pH}$ the relation between rate and buffer concentration was linear. With the morpholine buffers, the plot of rate against buffer concentration gave a somewhat curved line, the reason for which, however, was not discussed. The concentration range of the buffers used by him was much smaller than the range used by us.

At the start of this investigation, it was apparent to us that the interpretation of rate data for trifluoroacetanilide given by Biechler and Taft Jr. could not be correct. The purpose of the present study was primarily to elucidate the mechanism of hydroxyl ion catalysed hydrolysis of anilides with acidic properties. As the investigation progressed, it became apparent that some species other than hydroxyl ions are very effective catalysts. Since it was considered important to obtain information about these catalysts, a considerable part of this study is devoted to the effectiveness and mechanism of action of catalysts other than hydroxyl ions.

\section{MATERIALS AND METHODS}

Materials. The trifluoroacetanilide was prepared from trifluoroacetic acid and aniline; $;^{2,4}$ m.p. $86^{\circ}$ (determined in an electrically heated metal block, using a calibrated Anschütz thermometer). Literature values vary between 86 and $90^{\circ} \mathrm{C}$. Equiv. weight (determined by non-aqueous titration with $0.1 \mathrm{M}$ tetrabutylammonium hydroxide, using pyridine as solvent and azoviolet as indicator) 187.8 , calc. 189.1. The trichloroacetanilide was prepared according to Votocek and Burda $;^{5}$ m.p. $95-97^{\circ} \mathrm{C}$ (Ref. 5 gives 95-97 ). Equiv.

\footnotetext{
* When this paper was ready for publication, the communication of Schowen, R. L. and Zuorick, G. M. (Tetrahedron Letters 43 (1965) 3839) about glycinate-catalysed hydrolysis of trifluoro- $N$-methyl-acetanilide came to our notice.
}

Acta Chem. Scand. 20 (1966) No. 7 
weight (non-aqueous titration) 238.1, calc. 238.5. The potassium chloride (reagent grade, Merck, Darmstadt) used for obtaining constant ionic strength, was for some experiments recrystallised twice from $0.1 \mathrm{M}$ hydrochloric acid.

Hydrolysis experiments and determination of acidity constants. The kinetic experiments, as well as the determinations of the acidity constants and of the ionic product of water were performed at $25 \pm 0.05^{\circ} \mathrm{C}$ in a medium of $9.6 \%(\mathrm{v} / \mathrm{v})$ ethanol and ionic strength 1 - obtained by addition of $\mathrm{KCl}$. All the kinetic experiments were performed at constant $\mathrm{pH}$ and the concentration of the anilides was generally about $0.001 \mathrm{M}$. Because of the restricted solubility of trichloroacetanilide, concentrations down to about $0.6 \times 10^{-4} \mathrm{M}$ were used at the lowest $\mathrm{pH}$ values. The experiments at the higher $\mathrm{pH}$ values were performed in volumetric flasks in a thermostated bath. At hydroxyl ion concentrations smaller than about $0.01 \mathrm{M}$, a $\mathrm{pH}$-stat method was used to maintain constant $\mathrm{pH}$. The reaction vessel was equipped with a magnetic stirrer and a jacket through which water from an ultra-thermostat was circulated. In both cases, samples were withdrawn and analysed after appropriate intervals. A Radiometer model pHM 4 equipped with a glass and a saturated calomel electrode was used both in the pH-stat method and in the titrations described below. The electrodes were standardised with $0.05 \mathrm{M}$ potassium biphthalate and $0.01 \mathrm{M}$ borax ${ }^{6}$ before and after each experiment. In all the experiments, the $\mathrm{pH}$ readings for the standard solutions before and after an experiment agreed well and the difference between the $\mathrm{pH}$ values of the standard solutions was close to the theoretical one (departure as a rule less than $0.02 \mathrm{pH}$ units). As soon as the pH changed by about 0.005 units, small amounts of sodium hydroxide solution were added by an Agla micro burette. Carbon dioxide was excluded by performing the experiments under nitrogen.

The relation between measured $\mathrm{pH}$ values and $\left[\mathrm{OH}^{-}\right]$and $\left[\mathrm{H}^{+}\right]$(concentration of hydroxyl and hydronium ions) was determined by measuring the $\mathrm{pH}$ of $100 \mathrm{ml}$ of $1 \mathrm{M}$ $\mathrm{KCl}$ in $9.6 \%(\mathrm{v} / \mathrm{v})$ ethanol upon addition of $1 \mathrm{M}$ sodium hydroxide or hydrochloric acid solutions. An Agla micro burette was used in these titrations as well as in the determinations of the acidity constants of the anilides. For values of $\left[\mathrm{OH}^{-}\right]$or $\left[\mathrm{H}^{+}\right]$between approximately $5 \times 10^{-4}$ and $2 \times 10^{-2} \mathrm{M}$, plots of $\log \left[\mathrm{OH}^{-}\right]$or $\log \left[\mathrm{H}^{+}\right]$against $\mathrm{pH}$ were linear with slopes of 1 and -1 , respectively. By extrapolation, the relationship between $\log \left[\mathrm{OH}^{-}\right]$or $\log \left[\mathrm{H}^{+}\right]$and the measured $\mathrm{pH}$ is obtained over the whole $\mathrm{pH}$ scale. From the intersection of the lines the $\mathrm{p} K_{\mathrm{w}}$ value of 13.92 is obtained. This value is in good agreement with what could be expected from the value obtained by Harned and Hamer ${ }^{7}$ in $1 \mathrm{M} \mathrm{KCl} \mathrm{(13.77)} \mathrm{and} \mathrm{from} \mathrm{the} \mathrm{known} \mathrm{influence} \mathrm{of} \mathrm{ethanol} \mathrm{on} \mathrm{the} \mathrm{ionization}$ product of water. ${ }^{8}$

Solutions of trifluoroacetanilide $(0.0011 \mathrm{M})$ and trichloroacetanilide $(0.0007 \mathrm{M})$ were titrated with $1 \mathrm{M}$ sodium hydroxide. In a typical such titration of trifluoroacetanilide, pH readings after 13 different additions of titrant between 10 and $70 \%$ of neutralisation gave the value of $9.51 \pm \mathbf{0 . 0 1}$ for the stoichiometric $\mathrm{p} K_{\mathrm{a}}$ and in a titration of trichloroacetanilide, 12 different additions between 15 and $60 \%$ of neutralisation gave the value of $9.98 \pm 0.01$. The hydrolysis taking place during titration up to these degrees of neutralisations is negligible. The constants have also been determined photometrically using a Beckman photometer model DK with thermostated cell compartment. The agreement between the constants obtained by the two methods is good.

Assay. Two different methods - here called the UV-method and the aniline-method have been used. In the UV-method, the withdrawn samples were added to a solution of hydrochloric acid in a volumetric flask and the volume adjusted by addition of water. The solutions to be measured were about $0.1 \mathrm{M}$ with respect to hydronium ions. The absorbance (A) of these acid solutions was read in 1-cm cells at the maximum absorbance of the unhydrolysed anilides, viz. at $242.5 \mathrm{~m} \mu$ with trifluoroacetanilide and at $248 \mathrm{~m} \mu$ with trichloroacetanilide. At these wave-lengths, the absorbance of the products of hydrolysis at infinite time $\left(\mathbf{A}_{\infty}\right)$ is almost negligible in acid solution. The pseudo firstorder rate constants were calculated from the slopes of the straight lines obtained when $\log \left(A-A_{\infty}\right)$ was plotted against time. The UV-method was used at hydroxyl ion concentrations of about $0.005 \mathrm{M}$ or higher. Using this method of analysis, the kinetic runs were followed for at least one half-life, and in most cases, for considerably longer periods. In the aniline method - used at hydroxyl ion concentrations smaller than about $0.005 \mathrm{M}$ - the aniline formed is diazotised and coupled with $N$-(1-naphthyl)ethylenediamine. The procedure used by Eriksson and $\mathrm{Omdal}^{8}$ in determinations of 2 -methylaniline was 
adopted with some modifications. During addition of the different reagents, nitrogen was bubbled through the reaction mixture. Aniline couples easier than 2-methylaniline and the addition of acetate buffer could therefore be omitted. $10 \mathrm{~min}$ after the addition of $N$-(1-naphthyl)ethylenediamine solution, $1 \mathbf{M}$ hydrochloric acid was added to give a total volume of 25,50 , or $100 \mathrm{ml}$. The absorbance was measured in 1 or $10 \mathrm{~cm}$ cells at $550 \mathrm{~m} \mu$, using a Beckman spectrophotometer model DU. A standard curve was made with freshly distilled aniline. The molar absorptivity is 47900 . From plots of the logarithm of residual anilide concentration or of aniline concentration (when the rate is very slow and the reaction followed only to about $4 \%$ hydrolysis or less) against time, the pseudo first-order rate constants were calculated. The plots always gave straight lines. In all kinetic runs, six to fourteen samples were withdrawn and analysed.

Formation of fluoride and chloride ions. Fluoride and chloride ions might also be formed during hydrolysis of the amide bond. If these ions are formed in appreciable amounts simultaneously with aniline, the rate of aniline formation might be influenced, especially after long reaction times. Therefore, in order to assess the rate at which these ions are formed, they were determined in partly and in completely hydrolysed solutions. Fluoride ions were estimated spectrophotometrically according to the method of Icken and Blank, ${ }^{10}$ which is based on the bleaching of a thorium-alizarin lake by fluoride ions. With the experimental conditions used, hydrolysis of about $0.2 \%$ of the fluorine is easily detected. No fluoride ions could be detected after complete hydrolysis of the amide bond at hydroxyl ion concentrations of 0.01 and $0.001 \mathrm{M}$. After acidification with nitric acid, chloride ions were determined electrometrically, using a Radiometer pHM 4 and the following cell:

$$
\mathrm{Ag}, \mathrm{AgCl} \text {; solution to be examined; } \mathrm{K}_{2} \mathrm{SO}_{4} \text { (sat); } \mathrm{Hg}_{2} \mathrm{SO}_{4}, \mathrm{Hg} \text {. }
$$

Emf-determinations of solutions containing known amounts of hydrochloric acid instead of trichloroacetanilide gave differences in emf close to the theoretical ones in the concentration range $10^{-4}$ to $10^{-2} \mathrm{M}$. By using an experimental curve relating the concentration of chloride ions to emf, concentrations down to about $10^{-5} \mathrm{M}$ could be roughly estimated. Some determinations were made on partly and on completely hydrolysed solutions with hydroxyl ion concentrations of $0.1,0.01$, and $0.001 \mathrm{M}$. These determinations showed that chloride ions were formed more rapidly from the ionised trichloroacetanilide than from ichloroacetate ions and that the concentration of chloride ions was small compared to $f:$ concentration of aniline both in partly and in almost completely hydrolysed solutions at ine higher concentrations of hydroxyl ions. At the lowest hydroxyl ion concentration, however, the concentrations of chloride ions were not negligible in comparison to those of aniline.

In all hydrolysis experiments with trichloroacetanilide, the solutions after being made alkaline assume a distinct odour of phenylisocyanide. This odour appears almost immediately in the strongly alkaline solutions and after some time in the most weakly alkaline solutions. Alkaline solutions containing aniline and sodium trichloroacetate $(0.001 \mathrm{M}$ with respect to both components) do not develop this odour even after a long time.

The mechanism of phenylisocyanide and chloride ion formation has not been investigated in detail but the conceivable influence of ionization of chlorine and of phenylisocyanide formation on the rate of aniline formation at low hydroxyl ion concentrations will be touched upon in the discussion.

\section{RATE EQUATIONS AND RESULTS OF HYDROLYSIS EXPERIMENTS}

The following reaction scheme explains the rate data obtained in pure hydroxyl ion catalysed hydrolysis and in hydrolysis catalysed by species giving a limiting value of the pseudo first-order rate constant $k_{\mathrm{obs}}$ at constant $\mathrm{pH}$. 


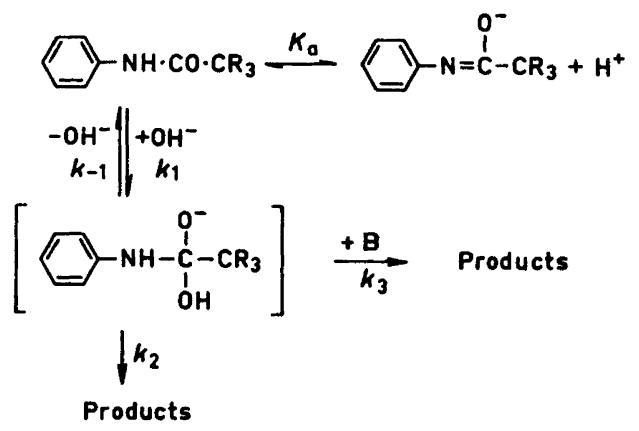

Assuming that the basic form of the anilide does not react, and applying the steady state approximation to the intermediate in brackets, henceforth called the intermediate, the following equation is derived:

$$
k_{\mathrm{obs}}=\frac{k_{1} K_{\mathrm{w}}}{K_{\mathrm{a}}+\left[\mathrm{H}^{+}\right]} \times \frac{k_{2}+k_{3, \mathrm{i}}\left[\mathrm{B}_{\mathrm{i}}\right]}{k_{-1}+k_{2}+k_{3, \mathrm{i}}\left[\mathrm{B}_{\mathrm{i}}\right]}
$$

Generally, B is hydroxyl ions or any other species catalysing the breakdown of the intermediate. If more than one species $B$ is operating in the breakdown of the intermediate, $k_{3, i}\left[\mathrm{~B}_{\mathrm{i}}\right]$ in eqn. (1) has to be replaced by $\sum k_{3, i}\left[\mathrm{~B}_{\mathrm{i}}\right]$. This is the case when, in addition to another catalyst operating in the breakdown process, the concentration of hydroxyl ions is large enough to cause a significant part of the hydrolytic reaction to proceed via $k_{3}$.

Figs. 1, 4, and 5 show that both compounds reach a limiting value of $k_{\text {obs }}$ at high concentrations of $\mathrm{B}$. When $k_{\text {obs }}$ does not increase with further increase in the concentration of $\mathrm{B}$, the whole reaction goes via $k_{3}$, as shown by the dotted curves in Figs. 1 and 4 . The value of $k_{3, i}\left[B_{i}\right]$ is then much larger than $\left(k_{-1}+k_{2}\right)$ and eqn. (1) can be simplified to

$$
k_{\mathrm{obs}}=\frac{k_{1} K_{\mathrm{w}}}{K_{\mathrm{a}}+\left[\mathrm{H}^{+}\right]}
$$

This equation shows that at limiting values of $k_{\mathrm{obs}}$, the rate determining step is formation of the intermediate and that $k_{1}$ is obtained from these values of $k_{\text {obs. }}$. If $\mathrm{B}$ is hydroxyl ions, $\left[\mathrm{H}^{+}\right]$according to Fig. 1 is small compared to $K_{\mathrm{a}}$ and eqn. (2) can then be written as

$$
k_{\mathrm{obs}}=k_{1} K_{\mathrm{w}} / K_{\mathrm{a}}
$$

At the lowest hydroxyl ion concentrations used in the experiments and with no catalytic species other than $\mathrm{OH}^{-}$present, the rate tends to become proportional to $\left[\mathrm{OH}^{-}\right]$. This being the case, $k_{2}$ must be much larger than $k_{3, \mathrm{OH}^{-}}\left[\mathrm{OH}^{-}\right]$and eqn. (1) can then be written as

$$
k_{\mathrm{obs}}=\frac{k_{1} K_{\mathrm{w}}}{K_{\mathrm{a}}+\left[\mathrm{H}^{+}\right]} \times \frac{k_{2}}{k_{-1}+k_{2}}
$$


or, if $K_{\mathrm{a}}$ is negligible compared to $\left[\mathrm{H}^{+}\right]$,

$$
k_{\mathrm{obs}}=\frac{k_{1} k_{2}\left[\mathrm{OH}^{-}\right]}{k_{-1}+k_{2}}
$$

With $k_{1}$ known, the quotient $k_{2} /\left(k_{-1}+k_{2}\right)$ or $k_{2} / k_{-1}$ can now be obtained from the experimental values at those small concentrations of hydroxyl ions, where rate is proportional, or almost so, to [ $\left.\mathrm{OH}^{-}\right]$.

From Figs, 1, 4, and 5 the concentration of $B$ at half the limiting values of $k_{\text {obs }}$ is obtained with rather good accuracy. In the case of pure hydroxyl ion catalysis, $\left[\mathrm{H}^{+}\right]$is negligible compared to $K_{\mathrm{a}}$ at this value of $k_{\text {obs }}$, and therefore the following expression is valid both when $B$ is hydroxyl ions (Fig. 1 ) and when $B$ is a catalyst used at constant pH (Figs. 4 and 5 ).

$$
k_{-1}-k_{2}=k_{3, \mathrm{i}}\left[\mathrm{B}_{\mathrm{i}}\right]
$$

Of course, curves of the type shown in Figs. 2 and 3 can also be used to estimate the relation between $\left(k_{-1}-k_{2}\right)$ and $k_{3, \mathrm{i}}$.

Knowing the values of $k_{2} / k_{-1}$ and of $k_{3, \mathrm{i}} /\left(k_{-1}-k_{2}\right)$ the quotients $k_{2} /\left(k_{-1}+k_{2}+\right.$ $\left.k_{3, \mathrm{i}}\left[\mathrm{B}_{\mathrm{i}}\right]\right)$ and $k_{3, \mathrm{i}}\left[\mathrm{B}_{\mathrm{i}}\right] /\left(k_{-1}+k_{2}+k_{3, i}\left[\mathrm{~B}_{\mathrm{i}}\right]\right)$ in eqn. (1) can be calculated at any concentration of $\mathrm{B}$, and all the parameters necessary for constructing profiles relating the value of $k_{\text {obs }}$ to the concentration of catalytic species are known. Knowing the value of $k_{2} / k_{-1}$, the values of $k_{3, i} / k_{-1}$, which are given in Tables 1 and 2 , are easily calculated from eqn. (6).

The rate equation proposed by Mader also contains a constant for the water-catalysed reaction. His rate profile indicates that this reaction is responsible for half the rate at the hydroxyl ion concentration of $10^{-6} \mathrm{M}$ and the computer analysis gave the value of $3.04 \times 10^{-5} \mathrm{~min}^{-1}$ for the rate constant. We have determined $k_{\text {obs }}$ in solutions acidified with hydrochloric acid to give hydrogen ion concentrations of $10^{-4}, 10^{-3}$, and $10^{-2} \mathrm{M}$. The medium was the same as that used in all the determinations of rate constants; the aniline formed was determined after appropriate intervals and the reactions were followed for several weeks. Owing to the very small degradations, the $\mathrm{pH}$-constancy was good. The following values for $k_{\text {obs }}$ were obtained:

$$
\begin{array}{ll}
\text { trifluoroacetanilide; } & 6.2 \times 10^{-7} \mathrm{~min}^{-1} \text { at }\left[\mathrm{H}^{+}\right]=10^{-3} \text { and } 10^{-4} \\
& 17 \times 10^{-7} \mathrm{~min}^{-1} \text { at }\left[\mathrm{H}^{+}\right]=10^{-2} \\
\text { trichloroacetanilide; } & 0.8 \times 10^{-7} \mathrm{~min}^{-1} \text { at }\left[\mathrm{H}^{+}\right]=10^{-3} \text { and } 10^{-4} \\
& 1.8 \times 10^{-7} \mathrm{~min}^{-1} \text { at }\left[\mathrm{H}^{+}\right]=10^{-2}
\end{array}
$$

That the rate is the same at hydronium ion concentrations of $10^{-3}$ and $10^{-4} \mathrm{M}$ and that it is much larger than the hydroxyl ion catalysed rate, strongly indicates that all hydrolysis taking place at these hydronium ion concentrations is water-catalysed and that the constants therefore are the pseudo monomolecular rate constants for water catalysis.

At the smallest concentrations of hydroxyl ions in Fig. 1, the values of the rate constants first obtained could not be repeated some months later. The smaller the hydroxyl ion concentrations were, the larger were the relative deviations. Potentiometric examination (titration with hydrochloric acid and with sodium hydroxide solution) of the potassium chloride used, revealed that different batches contained different amounts of impurities consuming

Acta Chem. Scand. 20 (1966) No. 7 


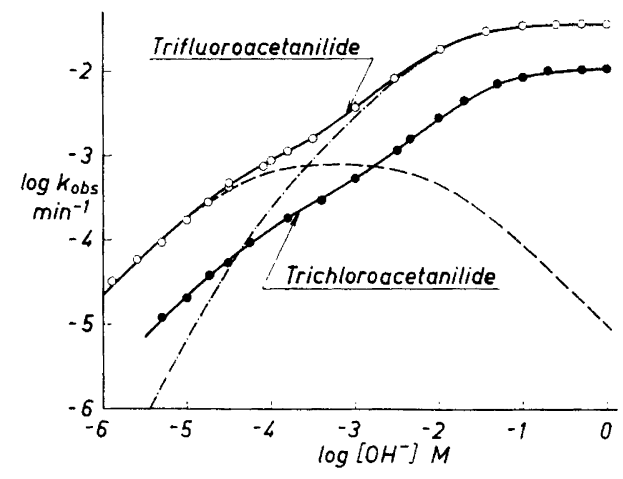

Fig. 1. Plot of $\log k_{\text {obs }}$ against $\log \left[\mathrm{OH}^{-}\right]$. The full lines are calculated from eqn. (1) and parameter values in Table 1 . For trifluoroacetanilide the dotted lines show the contribution to $k_{\text {obs }}$ from the reaction proceeding via $k_{2}(---)$ and via $k_{3}$ $(-\cdot-\cdot-)$. Experimentally determined values for trifluoroacetanilide $(0)$, and for trichloroacetanilide (O).

hydrogen ions. After recrystallisation twice from $0.1 \mathrm{M}$ hydrochloric acid and washing the crystals with ethanol, protolytic impurities could not be detected by potentiometric titration. Using this recrystallised potassium chloride, smaller values of the rate constants were obtained, which were also reproducible. Mader apparently obtained too large values for $k_{\text {obs }}$ at small concentrations of hydroxyl ions. One of the reasons might have been impurities in the potassium chloride. In order to get the experimental values to fit a calculated curve, the computer analysis gave him a large value of the constant for water-catalysis and a value of $K_{\mathrm{a}}$ that was twice as large as the photometrically determined value.

In Fig. $1, \log k_{\text {obs }}$ is plotted against $\log \left[\mathrm{OH}^{-}\right]$for trifluoro- and trichloroacetanilide. The curves are calculated from eqn. (1) and the parameter values used are presented in Table 1 . Most of the experimental values of $k_{\text {obs }}$ deviate by less than $5 \%\left(0.02 \mathrm{log}\right.$ units in $\left.k_{\text {obs }}\right)$ from the calculated ones and only exceptionally is the deviation as large as about $10 \%\left(0.04 \log\right.$ units in $\left.k_{\text {obs }}\right)$. For trifluoroacetanilide, the dotted lines show the contribution to $k_{\text {obs }}$ from the reaction proceeding via $k_{2}$ and $k_{3}$. From these lines it is apparent that at half the limiting value of $k_{\text {obs }}$ only about $2.5 \%$ of the hydrolysis proceeds via $k_{2}$. Also in the case of trichloroacetanilide, about $2.5 \%$ of the hydrolysis goes via $k_{2}$ at half the limiting value of $k_{\text {obs. }}$.

In the experiments with hydrogen carbonate or mono- and dihydrogen phosphate ions, presented in Figs. 2-5, these species at half the limiting

Table 1. Dissociation constants and rate parameters experimentally determined and used in the construction of Fig. 1 .

\begin{tabular}{|c|c|c|c|c|}
\hline Compound & $\mathrm{p} K_{\mathrm{a}}$ & $\begin{array}{c}k_{1} \\
1 \mathrm{~mole}^{-1} \mathrm{~min}^{-1}\end{array}$ & $k_{2} / k_{-1}$ & $\begin{array}{c}k_{3, \text { OH }} / k_{-1} \\
1 \mathrm{~mole}^{-1}\end{array}$ \\
\hline Trifluoroacetanilide & 9.51 & 930 & 0.025 & 93 \\
Trichloroacetanilide & 9.98 & 93 & 0.025 & 34 \\
\hline
\end{tabular}




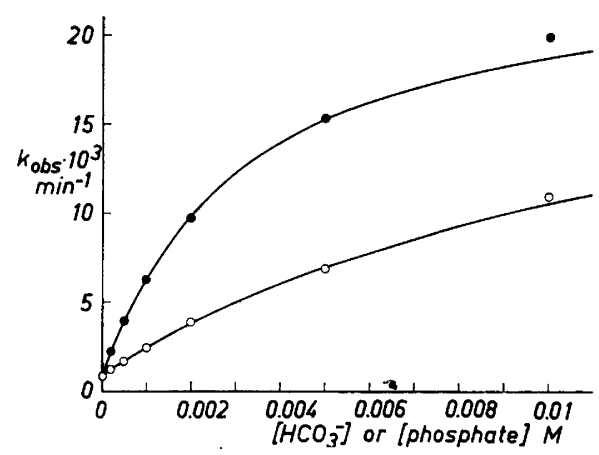

Fig. 2. Plot of $k_{\mathrm{obs}}$ for trifluoroacetanilide against $\left[\mathrm{HCO}_{3}^{-}\right]$and total concentration of phosphate. The lines are calculated from eqn. (1) and parameter values in Tables 1 and 2. Catalysis by $\mathrm{HCO}_{3}^{-}$at $\left[\mathrm{OH}^{-}\right]=$ 10-4.09 M (O), and by phosphate at $\left[\mathrm{OH}^{-}\right]=10^{-4.00} \mathrm{M}(\mathrm{O})$.

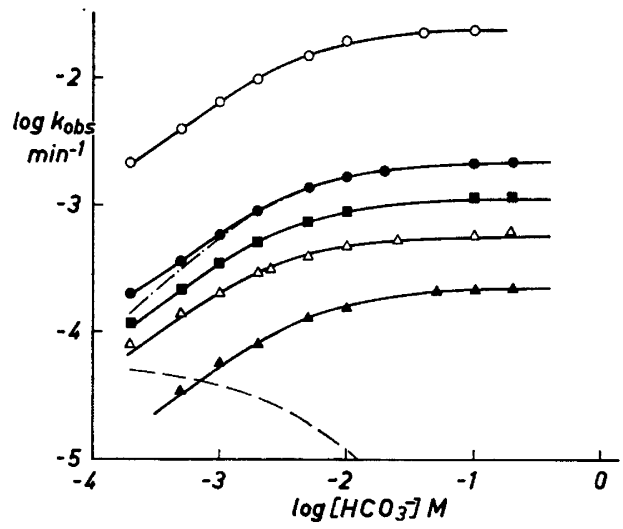

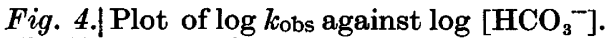
The lines are calculated from eqn. (1) and parameter values in Tables 1 and 2 . For trifluoroacetanilide at $\left[\mathrm{OH}^{-}\right]=10^{-5.6}$ the dotted lines show the contribution to $k_{\text {obs }}$ from the reaction proceeding via $k_{2}(---)$ and via $k_{3}(-\cdot-\cdot)$. Catalysis by $\mathrm{HCO}_{3}^{-}$at $\left[\mathrm{OH}^{-}\right]=10^{-5.60}$ for trichloroacetanilide $(\Delta)$, and for trifluoroacetanilide

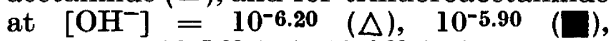
$10^{-5.60}(O), 10^{-4.09}(O)$.

Acta Chem. Scand. 20 (1966) No. 7

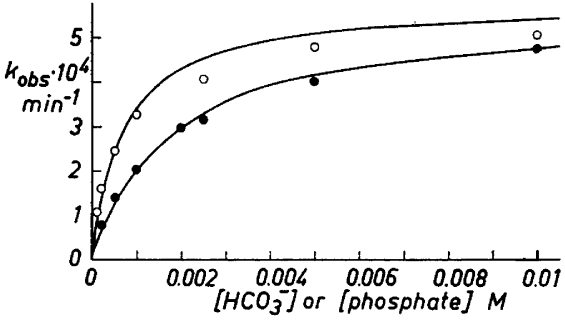

Fig. 3. Plot of $k_{\mathrm{obs}}$ for trifluoroacetanilide against $\left[\mathrm{HCO}_{3}^{-}\right]$and total concentration of phosphate. The lines are calculated from eqn. (1) and parameter values in Tables 1 and 2. Catalysis at $\left[\mathrm{OH}^{-}\right]=10^{-6.20} \mathrm{M}$ by $\mathrm{HCO}_{3}^{-}(\mathrm{O})$, and by phosphate (O).

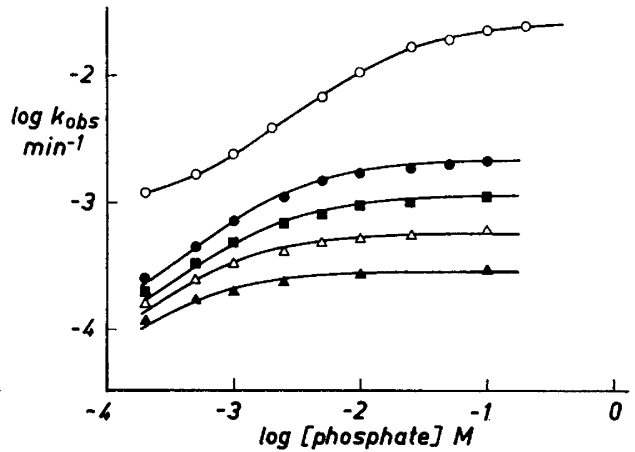

Fig. 5. Plot of $\log k$ obs for trifluoroacetanilide against log of total concentration of phosphate. The lines are calculated from eqn. (1) and parameter values in Tables 1 and 2. Catalysis by phosphate at $\left[\mathrm{OH}^{-}\right]=10^{-6.50}(\Delta), 10^{-6.20}(\triangle)$, $10^{-5.90}(\square), 10^{-5.60}(\bigcirc)$, and $10^{-4.00}(\mathrm{O})$. 
value of $k_{\text {obs }}$ catalyse about $97.5 \%$ of the hydrolysis - as exemplified in Fig. 4 for hydrogen carbonate catalyses of trifluoroacetanilide at $\left[\mathrm{OH}^{-}\right]=$ $10^{-5.60} \mathrm{M}$. The deviations of the experimental values in Figs. 2-5 from the calculated ones are mainly of the same magnitude as just discussed for Fig. 1 .

Hydrogen carbonate and hydrogen phosphates are very effective catalysts. Fig. 2 illustrates the effect of small concentrations of these species at relatively high concentrations of hydroxyl ions, and Fig. 3 at a smaller one. A comparison of these plots also shows that in the $\mathrm{pH}$-range used, the relative enhancement of the hydroxyl ion catalysed rate in the case of hydrogen carbonate is almost constant for a given concentration of catalyst, whereas the effect of phosphate strongly increases when the ratio $\left[\mathrm{H}_{2} \mathrm{PO}_{4}^{-}\right] /\left[\mathrm{HPO}_{4}{ }^{-}\right]$is increased with decreasing $\mathrm{pH}$. In Figs. 4 and $5, \log k_{\text {obs }}$ is plotted against log $\left[\mathrm{HCO}_{3}^{-}\right]$or log [phosphate] for the experiments with hydrogen carbonate and phosphate ions as catalysts. The limiting values of $k_{\text {obs }}$ are calculated from eqn. (2), and $k_{3, i} / k_{-1}$ is estimated from half the limiting values of $k_{\text {obs }}$ in these plots. Using the values of $k_{3, i} / k_{-1}$ given in Table 2, the curves in Figs. 2-5 are calculated from eqn. (1).

The $\mathrm{p} K_{\mathrm{a}}$ values of hydrogen carbonate and dihydrogen phosphate in a medium containing $9.6 \%$ ethanol and of ionic strength 1 were determined from measurements of hydrogen ion concentrations of solutions with equal concentrations of hydrogen carbonate and carbonate or dihydrogen phosphate and hydrogen phosphate ions. The following values were obtained from $\mathrm{pH}$ measurements at total concentrations of acid and its conjugate base ranging from 0.1 to $0.002 \mathrm{M}$ : $\mathrm{p} K_{\mathrm{a}, \mathrm{HCO}_{3}}=9.81 \pm 0.03, \mathrm{p} K_{\mathrm{a}_{3} \mathrm{H}_{3} \mathrm{PO}_{4}^{-}}=6.52 \pm 0.03$. The highest concentration of hydroxyl ions used in experiments with hydrogen carbonate corresponds to equal concentrations of hydrogen carbonate and carbonate ions and the value of $k_{3, \mathrm{HCO}_{3}}-k_{-1}$ obtained in this experiment is not higher than the values at smaller hydroxyl ion concentrations, thus indicating that carbonate ions are not operating in the breakdown of the

Table 2. Rate parameters experimentally determined and used in the construction of Figs. 2-6. The calcd. values of $k_{3, \mathrm{~B}} / k_{-1}$ are obtained from eqn. (7) using the following

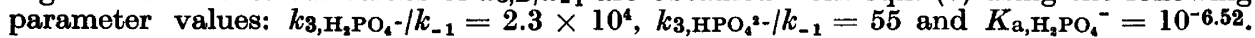

\begin{tabular}{|c|c|c|c|c|}
\hline Compound & $\begin{array}{c}\text { Catalytic } \\
\text { species } \\
\text { B }\end{array}$ & $\log \left[\mathrm{OH}^{-}\right]$ & $\log _{1 \mathrm{~mole}^{-1}}\left(k 3, \mathrm{~B} / k_{-1}\right)$ & $\begin{array}{c}\log \left(k_{3, \mathrm{~B}} / k_{-1}\right) \\
\text { calc. } \\
1 \mathrm{~mole}^{-1}\end{array}$ \\
\hline $\begin{array}{c}\text { Trifluoroacetanilide } \\
\text { Trichloroacetanilide } \\
\text { Trifluoroacetanilide } \\
\text { " } \\
\text { " }\end{array}$ & $\begin{array}{c}\mathrm{HCO}_{3}^{-} \\
" \\
" \\
" \\
\mathrm{H}_{2} \mathrm{PO}_{4}^{\prime-} \text { and } \\
\mathrm{HPO}_{4}{ }^{-2} \\
" \\
" \\
" \\
"\end{array}$ & $\begin{array}{l}-6.20 \\
-5.90 \\
-5.60 \\
-4.09 \\
-5.60 \\
-6.50 \\
-6.20 \\
-5.90 \\
-5.60 \\
-4.00\end{array}$ & $\begin{array}{l}2.71 \\
2.61 \\
2.53 \\
2.51 \\
2.43 \\
\\
3.43 \\
3.16 \\
2.85 \\
2.66 \\
1.81\end{array}$ & $\begin{array}{l}3.42 \\
3.15 \\
2.88 \\
2.62 \\
1.81\end{array}$ \\
\hline
\end{tabular}




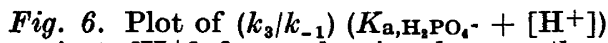

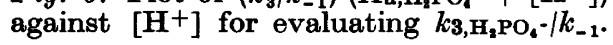

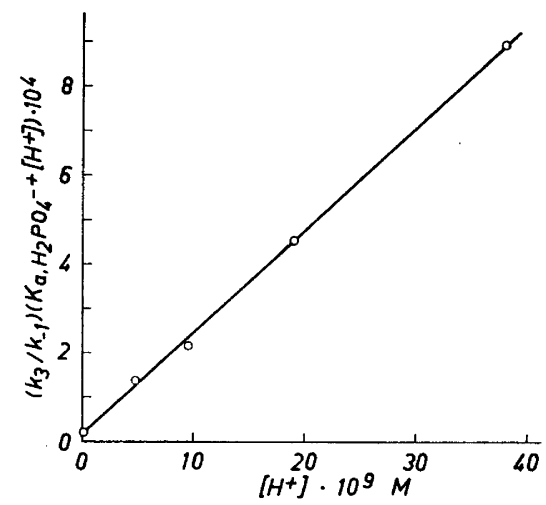

intermediate. In the case of phosphate catalysis, the value of $k_{3} / k_{-1}$ increases

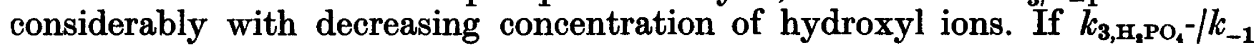
and $k_{3, \mathrm{HPO}_{2}-}-k_{-1}$ are the catalytic constants for the two species respectively, and if the phosphate is present only as $\mathrm{H}_{2} \mathrm{PO}_{4}^{-}$and $\mathrm{HPO}_{4}{ }^{2-}$, the following equation is valid

$$
\frac{k_{3}}{k_{-1}}=\frac{k_{3, \mathrm{H}_{3} \mathrm{PO}_{4}^{-}}}{k_{-1}} \times \frac{\left[\mathrm{H}^{+}\right]}{K_{\mathrm{a}}+\left[\mathrm{H}^{+}\right]}+\frac{k_{3, \mathrm{HPO}^{2-}}}{k_{-1}} \times \frac{K_{\mathrm{a}}}{K_{\mathrm{a}}+\left[\mathrm{H}^{+}\right]}
$$

where $k_{3} / k_{-1}$ is the experimentally determined catalytic parameter at different concentrations of hydroxyl ions and $K_{\mathrm{a}}$ is the acidity constant of $\mathrm{H}_{2} \mathrm{PO}_{4}^{-}$. Eqn. (7) can be written as

$$
\frac{k_{3}}{k_{-1}}\left(K_{\mathrm{a}}+\left[\mathrm{H}^{+}\right]\right)=\frac{k_{3, \mathrm{H}_{2} \mathrm{PO}_{4}^{-}}}{k_{-1}} \cdot\left[\mathrm{H}^{+}\right]+\frac{k_{3, \mathrm{HPO}_{4}^{2-}}}{k_{-1}} \cdot K_{\mathrm{a}}
$$

In Fig. 6, $\left(k_{3} / k_{-1}\right)\left(K_{\mathrm{a}}+\left[\mathrm{H}^{+}\right]\right)$is plotted against $\left[\mathrm{H}^{+}\right]$. From the slope of this line, the value of $2.3 \times 10^{4} 1 \mathrm{~mole}^{-1}$ is obtained for $k_{3, \mathrm{H}_{2} \mathrm{PO}_{4}-1 k_{-1}}$. At $\left[\mathrm{OH}^{-}\right]=10^{-4}$, most of the catalytic effect is brought about by $\mathrm{HPO}_{4}^{2-}$ and the value of $541 \mathrm{~mole}^{-1}$ for the parameter $k_{3, \mathrm{HPO}}{ }^{2-}-1 k_{-1}$ reported in Table 2 is calculated from eqn. (7) and from the experimental value of $k_{3} / k_{-1}$ when $\left[\mathrm{OH}^{-}\right]=10^{-4} \mathrm{M}$.

\section{DISCUSSION}

In his reaction scheme, Mader postulated formation of the intermediate<smiles>[O-]C(O)(Nc1ccccc1)C(F)(F)F</smiles>

through hydration of the anion of trifluoroacetanilide, and he also discussed its potential formation through ionization of hydrated trifluoroacetanilide. These two pathways by which the intermediate may arise are, however, Acta Chem. Scand. 20 (1966) No. 7 
kinetically indistinguishable from its formation via $k_{1}$. Using the parameter values of Table 1 , the second order rate constant for alkaline hydrolysis of undissociated trifluoroacetanilide is calculated from the expression $k_{1} k_{2} /\left(k_{-1}\right.$ $+k_{2}$ ), giving a value of $23 \mathrm{l} \mathrm{mole}^{-1} \mathrm{~min}^{-1}$. According to Biechler and Taft Jr., this constant for trifluoro- $N$-methylacetanilide at $25.5^{\circ} \mathrm{C}$ and ionic strength 0.6 has the value of $147 \mathrm{l} \mathrm{mole}^{-1} \mathrm{~min}^{-1}$. This compound cannot give an intermediate through hydration of a conjugate base. For the ions $\mathrm{C}_{6} \mathrm{H}_{5} \mathrm{NHCOCH}_{2} \mathrm{~N}^{+}$ $\left(\mathrm{CH}_{3}\right)_{3}$ and $\mathrm{C}_{6} \mathrm{H}_{5} \mathrm{~N}\left(\mathrm{CH}_{3}\right) \mathrm{COCH}_{2} \mathrm{~N}^{+}\left(\mathrm{CH}_{3}\right)_{3}$, the corresponding constants are 0.0022 (determined in this laboratory) and $0.0073 \mathrm{l} \mathrm{mole}^{-1} \min ^{-1}$ (Ref. 1), resp., whilst for acetanilide and $N$-methylacetanilide at approximately $100^{\circ} \mathrm{C}$, Davis ${ }^{11}$ found the values of 0.053 and $0.0641 \mathrm{~mole}^{-1} \mathrm{~min}^{-1}$, resp. Compared with these two pairs of constants and with the constant for $N$-methyltrifluoroacetanilide, the constant for trifluoroacetanilide is not unexpectedly high and its value at least does not indicate significant formation of the intermediate through hydration of the anion.

The second order rate constant for $N$-methyltrifluoroacetanilide is of the order to be expected from polar and steric substituent constants.,12 If significant formation of the intermediate had taken place through hydration and ionization of this compound or of the acidic form of trifluoroacetanilide, abnormally high values of the rate constants should have been obtained. This not being the case is an indication that hydration of the carbonyl group is not an important step in the hydrolytic breakdown. ${ }^{13}$

From parameter values in Table 1 , the second order rate constant for undissociated trichloroacetanilide is found to have the value of $2.31 \mathrm{~mole}^{-1}$ $\mathrm{min}^{-1}$, which is one tenth of that for trifluoroacetanilide. A comparison of the parameter values in Table 1 shows that the larger value for the trifluoro derivative is to be attributed entirely to differences in $k_{1}$, because $k_{2} / k_{-1}$ has the same value for both compounds. In their investigation on the alkaline hydrolysis of acetanilide and its $p$-chloro, $p$-methyl, and $p$-methoxy derivatives, Bender and Thomas ${ }^{14}$ discussed some different pathways for the breakdown of the intermediate. The process favoured by them involves formation of a dipolar anion before breakdown to products. This hypothesis will be further discussed in connection with the proposed mechanisms for the action of catalytic species in the breakdown of the intermediate.

The processes leading to products from the intermediate via $k_{2}$ might according to Bender and Thomas be depicted by

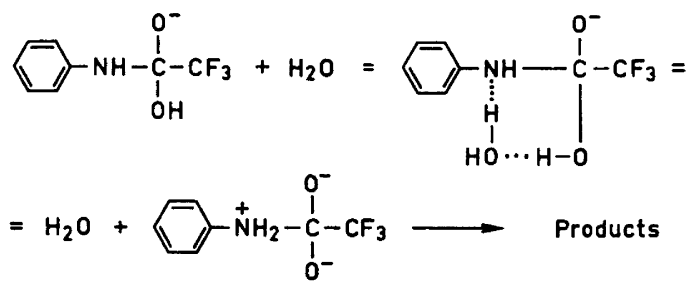


The dipolar anion rapidly breaks down to the stable fragments aniline and trifluoroacetate ion. Formation of the intermediate is, of course, facilitated by electron-attracting groups, and the difference in $k_{1}$ of the two compounds is therefore expected. From isotopic exchange studies, Bender and Thomas found that exchange was favoured over hydrolysis by electron-attracting groups in the para position, that is $k_{2} / k_{-1}$ decreases with the electron-attracting power of the substituents in the benzene nucleus. They also found that the effect of substituents on the rate of hydrolysis was very small, because the influence on $k_{1}$ was counteracted by the influence on $k_{2} / k_{-1}$. According to Bender and Thomas, the equilibrium position of dipolar anion formation essentially depends on the basicity of the nitrogen atom of the anilide and therefore the ratio $k_{2} / k_{-1}$ increases with the electron-donating power of the substituents in the benzene nucleus. However, the facility with which dipolar anions are formed also must be influenced by the ability of the hydroxyl group of the intermediate to donate a proton. For the two compounds investigated by us, the ratio $k_{2} / k_{-1}$ is the same and smaller than for any of the compounds investigated by Bender and Thomas. In contrast to substituents in the nucleus, substituents in the acylic part certainly affect the electron density in the hydroxyl group of the intermediate as much as at the nitrogen atom and therefore the same value of $k_{2} / k_{-1}$ for the trifluoro and trichloro compound does not oppose the theory of dipolar anion formation before breakdown to products.

For both compounds, $k_{3, \mathrm{OH}^{-}} / k_{-1}$ is much larger than $k_{2} / k_{-1}$. If $k_{2}$ is expressed as a second order rate constant for the reaction between intermediate and water, it is easily shown that hydroxyl ions are about $2 \times 10^{5}$ (trifluoroacetanilide) and $7 \times 10^{4}$ (trichloroacetanilide) times more effective than water molecules in the breakdown of the intermediate. These ratios are larger than those obtained by Biechler and Taft Jr. for $N$-methyltrifluoroacetanilide (about $10^{4}$ ) and some other substituted $N$-methylacetanilides. On the whole, their data also show that the attack of the second hydroxyl ion is strongly promoted by increasing electron-attracting power of the substituents.

Solutions of trichloroacetanilide completely hydrolysed at hydroxyl ion concentrations of $0.1,0.01$, and $0.001 \mathrm{M}$ all gave UV-spectra identical, or almost so, with the spectrum of aniline. The UV-spectra of aniline and phenylisocyanide in ethanol are rather different. ${ }^{15}$ Isocyanides are regarded as stable in alkaline solution and hydrolysable in acid solution. Since, however, quantitative data are lacking, it cannot be entirely excluded that some of the phenylisocyanide formed might have been hydrolysed to aniline also in alkaline solutions. In the hydrolysis experiments at small hydroxyl ion concentrations, the aniline was assayed by the method involving acidification followed immediately by diazotisation (reaction time $3 \mathrm{~min}$ ). After the diazotisation, isocyanide apparently remained in the reaction mixture, as evidenced by its characteristic odour. With a long time-interval between acidification and diazotisation, the odour disappeared without, however, a measurable increase in the aniline content being observed. If the amounts of aniline formed from phenylisocyanide are not negligible in comparison to the amounts of aniline primarily formed, the values of $k_{\text {obs }}$ will not correspond to pure anilide hydrolysis. Chloride ions might be formed without formation of phenylisocyanide but it is hard

Acta Chem. Scand. 20 (1966) No. 7 
to visualise a mechanism for formation of phenylisocyanide without simultaneous formation of chloride ions. Therefore, it can be concluded from the determinations of chloride ion that at high concentrations of hydroxyl ions the rate of formation of phenylisocyanide is negligible compared to the rate of formation of aniline. No such conclusion can be drawn regarding the rates at small hydroxyl ion concentrations. At small hydroxyl ion concentrations, the values of $k_{\text {obs }}$ have always been calculated from initial rates and therefore it can be assumed that products other than phenylisocyanide formed simultaneously with chloride ions do not significantly affect the determined rates of aniline formation. The facts discussed above together with the good agreement of the experimental values with the theoretically calculated $\mathrm{pH}$-profile, and the uniformity of the profiles of the two compounds indicate that also at small concentrations of hydroxyl ions most of the aniline is formed via direct hydrolysis of the amide bond.

The postulated mechanism with the catalytic species acting in the breakdown of the intermediate only, explains all the results obtained with hydrogen carbonate and phosphate ions. Formation of a complex between the anilides and the catalytic species would also lead to a hydrolytic behaviour of the kind actually obtained if the complex were hydrolysed much more rapidly than the free anilide; the limiting rate should then correspond to complete complex formation. Using four different concentrations of trifluoroacetanilide $\left(0.513 \times 10^{-3}, 1.026 \times 10^{-3}, 2.056+10^{-3}\right.$, and $\left.4.466 \times 10^{-3} \mathrm{M}\right)$, the same hydroxyl ion concentration $\left(10^{-5.6} \mathrm{M}\right)$ and the same hydrogen carbonate concentration $(0.001 \mathrm{M})$ in all the experiments, the rate constants $k_{\text {obs }}$ were determined. In order of increasing anilide concentration, the following values were obtained: $6.08 \times 10^{-4}, 6.00 \times 10^{-4}, 6.25 \times 10^{-4}$, and $5.91 \times 10^{-4} \mathrm{~min}^{-1}$. These values show no trend depending on substrate concentration. If complex formation were responsibe for the hydrolytic behaviour found, small concentrations of anilide in this case should have given a comparatively larger complex concentration and larger values of $k_{\text {obs. }}$. This not being the case, complex formation between substrate and catalytic species must be excluded as an explanation for the results obtained.

If an intermediate were formed in a reaction between anilide and hydrogen carbonate or phosphate ions and products were formed from this intermediate in a reaction with hydroxyl ions, there would be no reason for obtaining a limiting value of $k_{\text {obs }}$. Therefore, also this mechanism can be excluded. Generally, it is not likely that hydrogen carbonate and dihydrogen phosphate ions should act as strong nucleophilic catalysts, whereas carbonate and hydrogen phosphate ions should have no or a smaller catalytic effect.

The values of $k_{3, i} / k_{-1}$ in Table 2 show that hydrogen carbonate and particularly dihydrogen phosphate ions are much more effective than hydroxyl ions in the breakdown of the intermediate. A few preliminary and as yet unpublished hydrolysis experiments with trifluoroacetanilide and some other potential catalysts have been carried out and will be briefly discussed, because the results of these experiments assist in elucidating the proposed mechanism of hydrogen carbonate and phosphate catalysis. The catalytic effect of the hydrazinium ion $\mathrm{H}_{3} \mathrm{~N}^{+}-\mathrm{NH}_{2}$ seems to be of the same order of magnitude as that of the hydrogen carbonate ion, whereas acetate and citrate ions show 
only very small effects. Buffers of ammonia, morpholine, and monoethanolamine act in the breakdown of the intermediate but they also seem to attack the substrate directly, because at a given $\mathrm{pH}$, rates higher than the limiting rates calculated from eqn. (2) are obtained at high ammonia or amine concentrations. In small concentrations, these buffer species mainly catalyse the breakdown of the intermediate, but they are not at all as effective as hydrogen carbonate and dihydrogen phosphate ions. Triethanolamine buffers catalyse the hydrolysis significantly but they seem to leave the intermediate intact, because these buffers give, as also found by Mader, linear relationships between rate and concentration of catalyst.

The experiments with ammonia, morpholine, and monoethanolamine buffers at different $\mathrm{pH}$ values indicate that breakdown of the intermediate is catalysed chiefly by the acidic form, whereas the basic form acts as a nucleophilic catalyst on the substrate directly. Triethanolamine seems to act only in the last mentioned manner. In the case of hydrogen carbonate, the phosphates, and the hydrazinium ion it is easy to visualise a mechanism where sterically-favoured transition states precede formation of dipolar anions and breakdown to products in a reaction sequence, that, exemplified by trifluoroacetanilide and hydrogen carbonate, can be formulated by

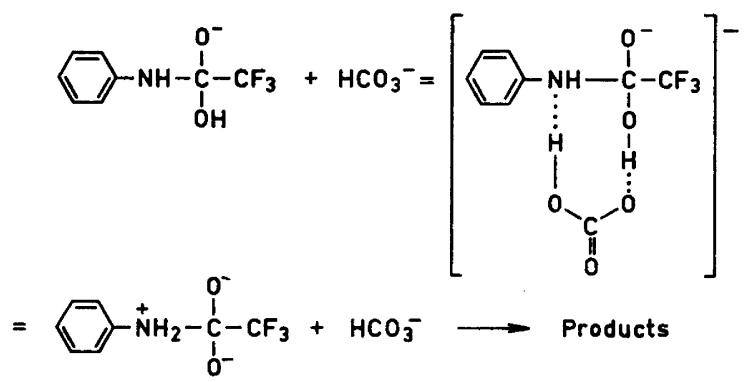

The simultaneous acceptance and release of protons seem to be at least possible with these catalysts; the hydroxyl ion, on the other hand, probably initiates breakdown of the intermediate through withdrawal of a proton from the latter's hydroxyl group ( $\mathrm{OH}^{-}$acting as a general base), whilst the ammonium ion and the positively charged amines seem to act by donating a proton to the intermediate's anilide nitrogen (the cations acting as general acids). In the case of the bulky triethanolammonium ion, the hydrogen atom at the nitrogen certainly can approach the anilide nitrogen only with great difficulty. The somewhat larger values of $k_{3, \mathrm{HCO}_{3}}-/ k_{-1}$ for the hydrogen carbonate catalysed experiments at the smallest hydroxyl ion concentrations might indicate that free carbonic acid is more effective than hydrogen carbonate ions in the breakdown of the intermediate. The interpretation of phosphate catalysis leading to the result that dihydrogen phosphate ions are much more effective than hydrogen phosphate ions seems reasonable. In hydrogen phosphate ion catalysis the presumed transition state acquires a treble negative net charge and its formation probably is less favoured than transition states with a smaller negative net charge. That the dihydrogen

Acta Chem. Scand. 20 (1966) No. 7. 
phosphate ion is a much stronger acid than the hydrogen phosphate ion also might be of importance.

Acknowledgements. We wish to thank Dr. Allan Agren for his interest in this work and to acknowledge the skilful technical assistance given by Mrs. Berit Jansson and Miss Susi Burkhart. Professor Richard Dahlbom read the manuscript and gave some valuable comments on it. A grant from Anslaget för ograduerade forskares vetenskapliga verksamhet vid Kungliga Farmaceutiska Institutet to one of us (S.O.E.) is gratefully acknowledged.

\section{REFERENCES}

1. Biechler, S. S. and Taft, Jr., R. W. J. Am. Chem. Soc. 79 (1957) 4927.

2. Bourne, E. J., Henry, S. H., Tatlow, C. E. M. and Tatlow, J. C. J. Chem. Soc. 1952 4014.

3. Mader, P. M. J. Am. Chem. Soc. 87 (1965) 3191.

4. Swarts, F. Bull. Sci. Acad. Roy. Belg. 8 (1922) 343.

5. Votocek, E. and Burda, J. Ber. 48 (1915) 1002.

6. Bates, R. G. Determination of $p H$, Wiley, New York 1964, p. 76.

7. Harned, H. S. and Hamer, W. J. J. Am. Chem. Soc. 55 (1933) 2194.

8. Gutbezahl, B. and Grunwald, E. J. Am. Chem. Soc. 75 (1953) 565.

9. Eriksson, S. O. and Omdal, J. O. Acta Pharm. Suecica 1 (1964) 77.

10. Icken, J. M. and Blank, B. M. Anal. Chem. 25 (1953) 1741.

11. Davis, O. C. M. J. Chem. Soc. 95 (1909) 1397.

12. Taft, Jr., R. W. In Newman, M. S. Steric Effects in Organic Chemistry, Wiley, New York 1956.

13. Bender, M. L. J. Am. Chem. Soc. 75 (1953) 5986.

14. Bender, M. L. and Thomas, R. J. J. Am. Chem. Soc. 83 (1961) 4183.

15. Ugi, I. and Meyr, R. Chem. Ber. 93 (1960) 239.

Received March 30, 1966. 